\title{
Shadow IT como Ferramenta Educacional: Uma Abordagem no Ensino Superior
}

\author{
Fernando Rodrigues, Wesley B. Thereza, Darley D. de Almeida, Uilliam Oliveira
}

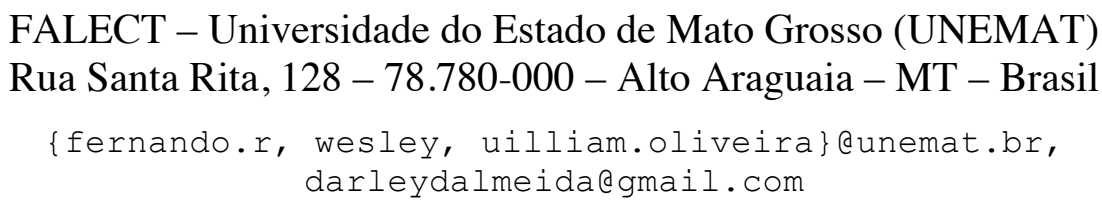

\begin{abstract}
Information Technology area (I.T.) has been changing due to the technologies that professionals have brought into the organizations without the knowledge of this science. Thus arises the term Shadow IT which is related to the use of any hardware and software tha is used to manipulate organizational data and I.T. Department is unaware. The purpose of this paper is to verify if Shadow IT is used in educational environment. For this, a bibliographic survey on Shadow IT was performed, as well as an investigation in the area of computer science in education, in order to establish the relationship between the use of hardware and software - that do not belonged to the Institution - by students, to learn in Higher Education context.
\end{abstract}

Resumo. A área de Tecnologia da Informação (T.I.) tem se modificado devido às tecnologias que os profissionais têm trazido para o interior das organizações sem o conhecimento dessa ciência. Assim, surge o termo Shadow IT que está relacionado à utilização de qualquer hardware e software que são usados para manipular dados da organização e o T.I. não tem conhecimento. O objetivo do presente trabalho é verificar se há o uso de Shadow IT no ambiente educacional. Para isso, foi realizado um levantamento bibliográfico sobre Shadow IT, além de uma investigação na área da informática na educação, a fim de estabelecer a relação entre o uso pelos alunos de hardware e softwares que não pertencem a Instituição, para se ter o aprendizado no âmbito do Ensino Superior.

\section{Introdução}

O papel do departamento de Tecnologia da Informação (T.I.) das empresas tem se modificado nos últimos anos. Dentre os vários motivos, destaca-se o uso de ferramentas de software e hardware pelos funcionários, sem que o próprio T.I. tenha conhecimento. Esta tendência vem aumentando devido aos fenômenos de Computação em Nuvem e consumerização de T.I., facilitando a utilização das tecnologias trazidas pelos funcionários, sem precisar de ajuda do departamento de T.I. Surgiu neste ambiente o Shadow IT, também conhecido como T.I. Invisível ou T.I. das Sombras (MALLMANN, 2016).

O termo Shadow IT é qualquer hardware e software utilizado pelos usuários sem conhecimento do departamento de T.I., ou seja, usar aplicativos e dispositivos para acessar e manipular dados da empresa sem conhecimento do T.I. (RENTROP E ZIMMERMANN 2012; GYÖRY et al. 2012; WALTERS 2013; SILIC E BACK 2014; GOZMAN 2015; HUBER et al. 2016; ALMEIDA et al. 2017; MALLMANN 2018). 
O Shadow IT vem aumentado no mercado, proporcionando muitos desafios e mudanças nos negócios tradicionais, entretanto há pouca informação documentada na área da educação, que também conta com um gerenciamento de T.I. das instituições. Ao analisar esse cenário, foram levantadas algumas perguntas:

- O Shadow IT existe dentro de ambientes educacionais?

- Caso ocorra o Shadow IT no ambiente educacional, levanta-se uma segunda hipótese: de que maneira ocorre?

- As pessoas sabem o que é Shadow IT?

A justificativa para o presente trabalho fundamenta-se em razão da necessidade de aprender e disseminar conhecimento sobre o Shadow IT, sendo um tema pouco explorado, principalmente quando aplicado na área educacional. A abordagem Shadow IT não se trata de um conceito novo, é um fenômeno com interpretação distorcida e pouco explorado de acordo com Silic e Back (2014).

Segundo o site CIO (2015), BT Global Services, empresa de telecomunicações do Reino Unido, "No Brasil, a média do Shadow IT responde atualmente por 32\% dos gastos com T.I., contra $25 \%$ da média internacional". A confiabilidade dos departamentos vem aumentando de forma exponencial na compra de suas próprias soluções de T.I., mudando o foco dos CIOs das companhias em geral, que passa da prestação de suporte para um papel mais estratégico, ligado a consultoria, governança e segurança. Na verdade, atualmente, os CIOs atuantes no Brasil estão gastando $26 \%$ a mais com tempo e recursos na área de segurança como resultado de Shadow IT - contra uma média global de $20 \%$.

\section{Metodologia}

Em uma pesquisa bibliográfica realizada na ferramenta Google Acadêmico, utilizando as strings de busca "Shadow IT na educação", "education Shadow IT" e "Shadow IT", não trouxe qualquer resultado que contenha o uso de Shadow IT na área educacional.

Para solucionar as questões da pesquisa e atingir o objetivo geral proposto neste trabalho, o processo ocorreu em três fases: na primeira, efetuou-se o levantamento bibliográfico, análise e exploração sobre o termo Shadow IT; já na segunda realizou-se uma investigação bibliográfica sobre tecnologia, informática na educação e ensino a distância, em que pode ser verificada a utilização de dispositivos e aplicativos para o ensino e aprendizagem. E por fim, fora produzida a pesquisa de cunho quantitativo no Ensino Superior, sobre o uso de dispositivos e softwares por alunos em sala de aula, sem que o T.I. da Universidade tenha conhecimento.

\section{Resultados e Discussões sobre a Pesquisa}

A pesquisa ocorreu com o público-alvo sendo estudantes do curso de Ciência da Computação de uma Universidade estadual. No momento da pesquisa, registraram-se 119 matrículas de alunos no curso em questão, sendo que deste universo, a amostragem foi de trinta (30) discentes, ou seja, $25 \%$ do total de alunos, que concordaram em responder a pesquisa.

Este formulário foi compreendido por sete questões objetivas e uma questão dissertativa, totalizando então oito perguntas. 
Ao analisar as respostas às perguntas do questionário, é perceptível que os alunos utilizam recursos próprios, sejam de software ou hardware, para estudar, acompanhar as aulas, se comunicar, desenvolver programas, fazer anotações, ou editar apresentações. Dito isso, fica evidente o uso de Shadow IT na Educação.

\section{Considerações Finais e Trabalhos Futuros}

No decorrer da pesquisa encontraram-se respostas para as perguntas geradoras da mesma. Portanto, as respostas as duas primeiras perguntas se confirmaram positivamente pela maioria dos estudantes entrevistados, corroborando com o contexto atual de qualquer empresa. Além disso, eles não conhecem o termo Shadow IT.

É possível concluir que dentro de Instituições de Ensino Superior públicas pode ocorrer com frequência o uso de Shadow IT pelos alunos, sendo por meio da utilização notebooks, smartphones e softwares diversos.

Como trabalhos futuros, espera-se ampliar a pesquisa aplicando o questionário para alunos dos cursos de outras áreas que não sejam de tecnologia, a fim de comparar os resultados com os já obtidos no curso de Ciência da Computação. Essa comparação é necessária para verificar se os alunos dos cursos da área de Humanas também utilizam recursos tecnológicos (hardware e software) não prescritos pela Instituição para facilitar seus aprendizados em sala de aula.

\section{Referências}

ALMEIDA, F. et al. "Innovation through Shadow IT". 2017.

CIO. "Shadow IT já é uma realidade para $88 \%$ dos CIOs brasileiros, revela pesquisa. [S. 1.]”, 28 jan. 2015. Disponível em: <https://cio.com.br/shadow-it-ja-e-uma-realidadepara-88-dos-cios-brasileiros-revela-pesquisa/>. Acesso em: 12 abr. 2019.

GYÖRY, A.; CLEVEN, A.; UEBERNICKEL, F.; BRENNER, W. "Exploring the shadows: IT governance approaches to user-driver innovarion". European Conference on Information Systems (ECIS), 2012.

HUBER, M.; ZIMMERMANN, S.; RENTROP, C.; FELDEN, C. "The Relation of ShadowSystems and ERP Systems: Insights from a Multiple-Case Study”. Systems, v. 4, n. 1, p. 11, 2016.

MALLMANN, Gabriela Labres. "Antecedentes do comportamento de uso da Shadow IT e sua relação com o desempenho individual". 2016.

MALLMANN, Gabriela Labres; MAÇADA, Antônio Carlos Gastaud; ECKHARDT, Andreas. "We are social: A social influence perspective to investigate Shadow IT usage". In: Proceedings of the Twenty-Sixth European Conference on Information Systems (ECIS2018), Portsmouth, UK. 2018. p. 23-28.

RENTROP, C; ZIMMERMANN, S. "Shadow IT Management and Control of unofficial IT". ICDS: The Sixth International Conference on Digital Society Reference, 2012.

SILIC, M; BACK, A. "Shadow IT: A view from behind the curtain". Computers \& Security, Volume 45, Pages 274-283, 2014.

WALTERS, R. "Bringing IT out of the shadows". Network Security, Volume 2013, Issue 4, Pages 5-11, 2013. 